\title{
JACQUES DERRIDA, UN PLUS DE VIDA
}

\author{
Javier Pavez *
}

El texto propone una aproximación al pensamiento de la sobrevida [survie] en Jacques Derrida. A partir de su Introducción a 'El origen de la geometría' de Husserl, de 1962, hasta su última entrevista el 2004, o incluso en el texto que redactó para ser leído en su funeral, se pone en escena cierta insistencia de este cuasiconcepto, insistencia e intensidad que se despliega desde la cuestión de la escritura y lo se puede denominar economía de la muerte. El cuestionamiento de la teoría husserliana del Presente viviente, a través de la muerte, encuentra, así, en la "sobrevida", la afirmación de la vida: Un plus de vida.

Palabras Clave: Derrida - escritura - economía de la muerte - sobrevida afirmación - plus de vida.

JACQUES DERRIDA, A MORE THAN LIFE

The text proposes an approach to thinking of survival [survie] in Jacques Derrida. From his Introduction to Husserl's Origin of Geometry, of 1962, until his last interview in 2004, or even in the text written to be read at his funeral, it is staged certain insistence of this quasi-concept, insistence and intensity that unfolds from the question of the writing and from what can be called "economy of death". The questioning of Husserl's theory of the Living-present, through the reason of death, found, thus, in the survival, the affirmation of life: a more than life.

Keywords: Derrida - writing - economy of death - survival - affirmation - more than life.

* Correo electrónico: jvrpavez@gmail.com 


\section{Insistencia}

\section{La muerte por la escritura también inaugura la vida Jacques Derrida, De la gramatología.}

No SERÍA DESCAMINADO PROPONER QUE DURANTE la última década ha reaparecido con cierta potencia, en el contexto filosófico, la cuestión del pensamiento del concepto de vida. Sin duda es un asunto de larga data, cuyos alcances se arrastran desde Aristóteles, pero, sin negar la herencia, se podría reconocer que el amplio espectro de este retorno alcanza, con giros y cruces, el pensamiento de la biopolítica, de la animalidad, de la ontología, en problemáticas que involucran planteamientos en torno a Agamben, Canguilhem, Deleuze, Heidegger, Nietzsche, Simmel, entre otros ${ }^{1}$. Dentro de este contexto, proponemos una aproximación específica a este problema en el pensamiento de Jacques Derrida. Tal acercamiento es pertinente no sólo por el contexto y discusión filosófica en que se enmarca, sino que, igualmente, porque implica indagar acerca de lo que él designó como 'sobrevi-

1 El retorno de la cuestión vida podría tener, al mismo tiempo, la frágil tesitura de una insistencia. El registro de esta insistencia, plantea tanto divisiones como cruces, es decir, contaminaciones de orden teórico y práctico en los ámbitos ontológico, político y biológico. Respecto de esta última esfera -que no constituye un alveolo autónomo absolutamente distinto y determinado-, podría destacarse el texto de Françoise Jacob, La logique du vivant (Gallimard, Paris, 1970), texto sobre el cual Derrida presenta una lectura en su Seminario de 1974-75 La vie la mort (aún inédito), y sobre se puede rastrear un apunte en De la gramatología (p. 11 y ss) alrededor del concepto de "Pro-grama" (Cfr. Garrido, Juan Manuel, On Time, Being, and Hunger: Challenging the Traditional Way of Thinking Life, Hardcover Fordham University Press, pp. 71 y ss). Sobre este retorno en clave biológica, a su vez, remitimos al volumen colectivo editado por Françoise Monnoyeur, Questions Vitales. Vie biologique, vie psychique (Paris, Editions Kimé, 2009) así como al libro de Michel Morage Live explained (Yale University Press, New Haven, 2008) y su artículo "The resurrection of life" (en Origins of life and Evolution of Biospheres, No 40, 2010, pp. 179-182). Por otra parte, la republicación del libro de 1965 de George Canguilhem Knowledge of life (Fordham University Press, 2008), le da un nuevo aliento a sus trabajos, entre lo que cuentan el texto "Vie" publicado en la Encyclopaedia Universalis (Paris, Vol. 27, pp. 545-564). Tenemos que mencionar que la bibliografía de y sobre Nietzsche acerca de la vida y la muerte, es extensa, de la cual los libros de Vanessa Lemm: Nietzsche y el devenir de la vida (F.C.E., Santiago, 2014), The Government of Life: Foucault, Biopolitics and Neoliberalism (Fordham University Press, New York, 2014), Nietzsche and the Becoming of Life (Fordham University Press, New York, 2014), Nietzsche y el pensamiento político contemporáneo (F. C. E.. Santiago de Chile, 2013) y Nietzsche's Animal Philosophy: Culture, Politics and the Animality of the Human Being (Fordham University Press, New York, 2009), constituyen valiosos textos que a su vez dan cuenta de una amplia bibliografía complementaria. Muy cerca de Nietzsche, se abre el pensamiento de Deleuze acerca de la vida y la inmanencia (Cf. "La pensée et la vie" en Nietzsche et la philosophie, Paris, P.U.F., 1962, 114-115; "La literatura y la vida" en Crítica y clínica, Anagrama, Barcelona, 1996, pp. 11-18; y "La inmanencia: una vida" en Dos regímenes de locos, Pretextos, Barcelona, pp. 347-352), apuesta que recogen tanto Esposito, que, alrededor del devenir-animal, escribe acerca de una "biopolítica afirmativa" (Cf. Tercera persona. Política de la vida y filosofía de lo impersonal . 2007), como Agamben, que retoma el "una vida" de Deleuze y que podría leerse en una infinita aproximación con su planteamiento acerca de la potencia de una forma-de-vida, y de la consecuente distinción entre zoe y bios. En lo que atañe a esta distinción, y lo que se puede llamar un diferendo entre Agamben y Derrida, me permito remitir a mi ensayo "Vida espectral: biopolítica y desconstrucción" (en Actuel Marx intervenciones, No 18). 
da', bajo la hipótesis de que juega un rol decisivo en la formación y despliegue del pensamiento derridiano. La cuestión de la sobrevida requerirá, pues, revisar en sus trabajos cierta apuesta por la muerte para, justamente, afirmar la vida más allá del todo presente vivo, es decir, más allá de toda determinación de presencia. Veremos, así, que el sur de la survie, suplementa un exceso que afirma un plus de vida entendido a la vez como más que la vida y no más vida².

Si emprendemos una pesquisa de la variación de temáticas que este motivo sobrelleva, tendríamos que hacer notar que con justicia es el último al que Derrida hace mención en la carta que redactó para ser leída en su sepelio, donde exhortaba a preferir la vida y a afirmar sin descanso la sobrevida [survie] $]^{3}$ Lo último, aquí, no tiene la medida del relevo sino del retorno. Del giro y del torno, pues todo este motivo de la sobrevida compone un haz que podría -aprés coup - rastrearse desde aquella despedida leída por su hijo Pierre el 2004, hasta su Introducción a 'El origen de la geometría' de Husserl, de 1962, donde, alrededor del estatuto de los objetos ideales, ya se prefiguraba aquella última afirmación. Es decir, bajo la lógica de un ya intensivo ${ }^{4}$, la cuestión de la sobrevida se hacía lugar en esta temprana Introducción a través del pensamiento de la posibilidad de la escritura, en la medida en que asegura "la tradicionalización absoluta del objeto, su objetividad ideal absoluta, es decir, la pureza de su relación con una subjetividad trascendental universal"s A grandes rasgos, este texto gira en torno del problema de la conservación y transmisión histórica de los objetos ideales, es decir, de la comunicación en la tradición de las verdades (lógicas, por ejemplo). Si la idealidad es pura, si se pretende independiente de cualquier materialidad, ¿de qué modo interfiere la escritura y el lenguaje en general en la constitución de aquella idealidad? Sintéticamente: el medio de tradicionalización no es exterior a la idealidad, no es un vehículo instrumental sino que lo encuentra su condición en la inscripción material que la posibilita. De otra suerte dicho, asegurar la pureza de la relación de la objetividad ideal absoluta con una subjetividad trascendental universal, significa

2 Al mismo tiempo, creemos que resulta relevante nuestra tentativa pues el asunto de la sobrevida juega un rol decisivo el pensamiento derridiano, en el sentido que puede ofrecer una clave para rastrear en la génesis de sus conceptos, a través de esta contaminación entre la vida y la muerte, las implicancias que tocan el pensamiento sobre lo político, por ejemplo, la desconstrucción de la soberanía, así como la cuestión de la pena de muerte, pero también envite de la justicia y la democracia, toda vez que esta pregunta está marcado por el asunto del porvenir, que justamente aparece de manea particularmente estratégica desde sus primeros escritos.

3 Cfr. Derrida, Jacques, "Jacques Derrida", Rue Descartes, 2005/2 n 48, p. 6-7.

4 El "ya" (dejà), escribe DerRIDA, "por su 'forma' [...] y por su sentido, es un pasado más que pasado y más que presente-pasado [passé plus que passé et plus que présent-passé]" (Derrida, Jacques, "Ja o en la estacada" en El tiempo de una tesis: desconstrucción e implicaciones conceptuales, Prometeo, Barcelona, 1997, pp. 72-73). Toda esta temática del "más", del "plus", como veremos, estará en juego en lo que Derrida escribe acerca de la vida, de una vida cuyo porvenir no se deja pensar bajo la modulación de la presencia, del presente, sea entendido como presente-pasado o presente-futuro.

5 DerridA, Jacques, Introducción a 'El origen de la geometría' de Husserl, Manantial, Buenos Aires, 2000, p. 84. Sobre la cuestión del la historicidad como un motivo que atraviesa de cabo a rabo nuestro autor, desde sus primeros trabajos sobre Husserl hasta sus últimas reflexiones sobre "lo mesiánico", véase "La contamination et le retard: Phénoménologie de l'historicité et de la tradition chez Derrida" en Studia Phcenomenologica No, XI, 2011, pp. 195-220. Remitimos, también, a la tesis de Iván Trujillo, Fiction historique. Recherche sur le problème historique de la fiction dans la philosophie de Jacques Derrida, U. de Chile-U. Paris X, 2014. 
cuestionar la pureza y el carácter trascendental puro de la relación, porque expone que lo ideal no está asegurado de antemano. Lo desencadenado se encadena, lo incondicionado vive a condición de la inscripción que lo transmite, o la condición de posibilidad de lo excluyente es lo excluido, bien podría decirse acerca de este origen inscrito de la verdad. Esto quiere decir, a la vez, que las maneras de conservar y trasmitir lo ideal no son simplemente empíricas, sino que dan cuenta de un carácter trascendental de la constitución material. Cuasi-trascendetal, dirá posteriormente Derrida. Pero quedándonos en este texto, diríamos que la vida ideal de la verdad sólo es posible si sobrevive en la tradición. Lo que significa que la sobrevivencia, génesis de la idealidad, es la condición de la vida pura al mismo tiempo que es la encentadura que pone en cuestión la pretendida pureza.

A partir de este temprano trabajo, entonces, la cuestión de la escritura (y del retraso) le permite a Derrida poner en movimiento una deconstrucción, sin crítica (krinein), de la evidencia simple absoluta del Presente Viviente (Lebendige Gegenwart), de la presencia como forma originaria (Urform) del tiempo ${ }^{6}$. Así, la escritura "ya no es solamente el auxiliar mundano y mnemotécnico de una verdad cuyo sentido de ser prescindiría, en sí mismo, de toda consignación" sino que el "acto de escritura es, pues, la más alta posibilidad de toda 'constitución'. Y en esto se aprecia la profundidad trascendental de su historicidad"7. Cierta contaminación empírico/trascendental, está ya en juego a partir de la mencionada Introducción. Bien lo explica Françoise Dastur:

[...] la idealidad no está de ninguna manera antes o por encima de lo sensible, sino después o por debajo de ella, y no puede aparecer sino en aquello que podríamos ya denominar un modo de suplementariedad, su muerte en la facticidad es la condición de su nacimiento en tanto que idealidad, o como Derrida dirá más tarde, su condición de posibilidad que es al mismo tiempo la condición de su imposibilidad ${ }^{8}$.

Dicho sucintamente, la escritura pone en escena la temporalidad de la muerte en la dialéctica (protensión-retención) del Presente Viviente. Es en este sentido que "la escritu-

6 En este sentido, es considerable que El tiempo de una tesis (1980), Derrida explicite que su "Introducción" le permitió "una aproximación a algo así como la axiomática impensada de la fenomenología husserliana, de su 'principio de los principios', a saber el intuicionismo, el privilegio del presente viviente, la inatención al problema de su propia enunciación fenomenológica" detallando que "todos los problemas elaborados en la Introducción no han dejado de organizar las investigaciones que emprendí más adelante", por ejemplo, "la irreductibilidad de la alteridad que viene a dividir la identidad consigo del presente viviente." (El tiempo de una tesis. Deconstrucción e implicaciones conceptuales, Prometeo, Barcelona, 1997, pp. 14-15). No sólo aquí se insiste sobre esto. Años más tarde, en una declaración que toca la axiomática impensada, y esta vez en torno al animal, subraya Derrida que "siempre está lo impensado de un pensamiento de la vida" y añade que "por ahí, por la cuestión de la vida y del 'presente vivo', de la autobiografía del ego en su presente vivo, comenzó mi lectura deconstructiva de Husserl y, en verdad, todo lo que ha podido venir después" (El animal que luego estoy si(gui)endo, Trotta, Madrid, 2008, p. 187.).

7 Derrida, Jacques, Introducción... op. cit, p. 86. Sobre el "presente viviente" como "el movimiento de la conciencia originaria", "que no está en el tiempo pero que constituye el tiempo", Cfr., Thaо, Fenomenología y materialismo dialéctico, Lautaro, Buenos Aires, 1959, pp., 123-128, n. 6.

8 Dastur, Françoise, "Finitude et répétition chez Husserl et Derrida" en Alter. Revue de phénomenologie. $\mathrm{N}^{\circ}$ 8, 2000, p. 46. (La trad. es nuestra). 
ra como posibilidad y como condición intrínsecas de los actos de saber objetivo"9 pone radicalmente en cuestión la posición - la thesis - de la idealidad libre, así como la consiguiente oposición entre idealidad libre e idealidad encadenada (o idealidad cultural), y por lo tanto, la posición privilegiada de la vida trascendental. Esta puesta cuestión, por lo demás, se despliega incombustiblemente bajo cierta insistencia ${ }^{10}$. Bajo el registro de este envío intensivo, Derrida, hasta su último seminario, no dejará de mostrar que Husserl "determina su absoluto fenomenológico como Erlebenis, como vida, vida trascendental" "1 y de apostar por una "supervivencia que no es ni la vida ni la muerte pura y simple, ni pensable desde la oposición entre la vida y la muerte”, y de aventurar, así, un tejido "de pervivencia, como muerte en la vida o vida en la muerte, tejido que no viene a revestir una existencia más originaria, una vida o un cuerpo o un alma que existirían, desnudos, bajo esas ropas"12.

El pensamiento de la escritura, entonces, que afecta desde el interior el sentido de ser de la objetividad ideal, implica que la verdad ideal deba ser escrita para ser plenamente objetiva (ideal), implica que la perdurabilidad que posibilita la escritura es el sentido mismo de la verdad, es decir, que "las condiciones de su supervivencia [survie] se hallan implicadas en las de su vida"13. Si esto quiere decir que la idealidad no vive sino a condición de la inscripción que la posibilita, esto es, sino a condición de sobrevivir ¿Qué es esto sino afirmar la sobrevida? Así, la insistencia de la sobrevida-cuyo desencadenamiento arrastra el pensamiento de la escritura, del retraso, de la huella, de la différance- se arrastra a partir de la Introducción, hasta Espectros de Marx y más allá ${ }^{14}$. Es decir, mientras en el pensamiento de la fenomenología husserliana la "muerte no sería comprendida como sentido, sino como hecho extrínseco al movimiento de la temporalización"15, para Derrida el pensamiento de la archi-escritura "señala el tiempo muerto en la presencia del presente viviente, en la forma general de toda presencia"16. Como señala Dastur, nuestro

9 DERRIDA, Jacques, Introducción..., op. cit., p. 90.

10 "No pienso más que en la muerte; pienso siempre en ella; no pasan diez segundos sin que tenga presente su inminencia. Analizo constantemente el fenómeno de la supervivencia como estructura del sobrevivir; es en verdad la única cosa que me interesa, pero precisamente en la medida en que no creo en la supervivencia post mortem. A fin de cuentas, es esto lo que rige todo, todo cuanto hago, soy, escribo, digo." DerRiDA, Jacques, El gusto del secreto, Amorrortu, Buenos Aires, 2009.

11 Derrida, Jacques, Seminario La bestia y el soberano. Volumen II (2002-2003), Manantial, Buenos Aires, 2011, p. 167.

12 Ibidem, pp. 174-175.

13 DERRIDA, Jacques, Introducción., op. cit., p. 88.

14 En Espectros de Marx. El estado de la deuda, el trabajo del duelo y la nueva internacional (Trotta, Madrid, 2003) esta insistencia tendrá distintas modulaciones alrededor, por ejemplo, de la justicia que "debe llevar más allá de la vida presente" (p. 13), del sobre-vivir "cuya posibilidad viene de antemano a desquiciar o desajustar la identidad consigo del presente vivo así como de toda efectividad" (p. 14), de cierta acontecibilidad que resiste "la simple oposición (ideal, mecánica o dialéctica) de la presencia real del presente real o del presente vivo y de su simulacro fantasmático, en la oposición de lo efectivo (wirklicb) y lo no efectivo" (p. 84), o de cierto comunismo "que siempre está por venir y se distingue, como la democracia misma, de todo presente vivo como plenitud de la presencia a sí, como totalidad de una presencia efectivamente idéntica a sí misma" (p. 115).

15 DERRIDA, Jacques, Introducción.. op. cit., p. 144.

16 Derrida, Jacques, De la gramatología, Siglo XXI, México D. F. 1986, p. 88. En Ecografías de la televisión, DERRIDA escribe que el "duelo" y el "tormento" se "desencadenan antes de la muerte misma, a partir de la 
autor justamente va a "mostrar" que "Husserl no ve en la muerte la condición misma de la vida" ${ }^{17}$, estrategia de mostración que para nosotros, a través de una economía de la muerte, involucra el exceso [surplus] de un plus de vie.

\section{Economía de la muerte.}

Ya desde la Introducción... entonces se hace lugar cierto pensamiento de la contaminación (empírico-trascedental), y así, el pensamiento de la economía de la muerte, de la muerte en la vida. En esta dirección, por ejemplo, Paola Marrati subraya que "la contaminación de lo finito y lo infinito, de la vida y de la muerte es lo que se da a pensar en la noción derridiana de escritura"18. A partir de la escritura, dirá Marrati, hace falta pensar la sobrevida como la presencia de la vida siempre ya repetida, o la vida como siendo ya una forma de sobrevida, lo que "implicaría que la vida misma no es absolutamente viviente, sino que sabe de cierto trabajo de la muerte"19. La sobrevida, nombra, pues, este trabajo de muerte, la muerte en la vida, el exceso de vida como lo otro de la vida en la vida ${ }^{20}$. Es otro nombre, un sobrenombre del espaciamiento de la différance.

Indudablemente la vida se protege a sí misma mediante la repetición, la huella, la diferancia [différance]. Pero hay que tener cuidado con esa formulación: no hay vida primero presente, que a continuación llegase a protegerse, a aplazarse, a reservarse en

mera posibilidad de la muerte, es decir, de la traza que surge como inmediata super-vivencia" (Ecografías de la televisión. Entrevistas filmadas, Eudeba, Buenos Aires, 1998, p. 164). Así, la cuestión de la vida y su inexpugnable relación con la muerte, está de cabo a rabo atravesado por la alteridad, y conlleva una lectura del "duelo" como "duelo imposible". Sobre esto Cfr. Cragnolini, M. "Adieu Adieu. Remember me. Derrida, La escritura y la muerte" en Derrida, un pensador del resto, Buenos Aires, La Cebra, 2007, pp.35-46.

17 Dastur, Françoise, op. cit, p. 48.

18 Marrati, Paola, La genèse et la trace. Derrida lecteur de Husserl et Heidegger, Kluwer Academic Publishers, Dordrecht, 1998, p. 214 (Trad. nuestra). Ahora bien, la noción de escritura, su ejemplaridad, no se deja separar de la cuestión de la sobrevida: "La cuestión de la escritura es, a la vez, indisociable de la cuestión de la temporalidad: aquella concepción de la temporalidad nos permite pensar la experiencia de la escritura como sobrevida [survie]" (Ibídem, p. 145).

19 Ibídem, p. 60. Más adelante Marrati abordará el problema de la idealidad en Derrida como cierta "Idealidad sin presencia", que no es sino "el 'lugar' de la contaminación de la vida y de la muerte", lugar no es "ni apertura a la infinita presencia de la vida trascendental, ni finitud originaria del ser para la muerte", sino "idealidad como sobrevida". Así, vuelve a decir que "la escritura", "se ha impuesto, para Derrida, muy rápidamente como paradigma de aquella idealidad retornada", pues en la medida de que es "necesaria, según Husserl, para liberar la idealidad de toda dependencia de una comunidad empírica, gracias a su capacidad de funcionar en seco", vendría a "marcar esta contaminación de lo empírico y de lo trascendental que se da a leer en la necesidad de una inscripción mundana ahí donde el sentido intencional no se deja separar más del cuerpo del signo”, vendría a marcar el "valor testamentario" que "refleja tanto el 'duelo originario' como la sobrevida, al menos que no se trate de la mima cosa. Porque la escritura refleja la muerte siempre posible del escritor, gracias a su poder de sobrevivirle. Expuesta a la borradura, ella no promete una vida eterna, resta por un tiempo, sobrevive un poco" (Ibídem, 217-218).

20 “Tal como se definió (indefinidamente) en el pasaje de Le pas au-delà, el decreto o sentencia de muerte [l'arrêt de mort ] no solamente es la decisión que decreta o sentencia lo irresoluble [l'indécidable]: también detiene la muerte suspendiéndola, interrumpiéndola, retrasándola con un sobresalto, el sorprendente sobresalto del sobrevivir [sursaut d'une survie]. Pero entonces lo que suspende o mantiene la muerte a raya es la misma cosa que le da todo su poder de irresolubilidad -otro nombre falso, más que un seudónimo, para la diferencia [différance]." (Derrida, J., "Sobrevivir: Líneas al borde" en Deconstrucción y crítica, Siglo XXI México D. F., 2003 p. 114). 
la diferancia. Ésta constituye la esencia de la vida. Más bien, como la diferancia no es una esencia, como no es nada, no es tampoco la vida, si el ser se determina como ousia, presencia, esencialexistencia, sustancia o sujeto. Hay que pensar la vida como huella antes de determinar el ser como presencia. Esa es la única condición para poder decir que la vida es la muerte, que la repetición y el más allá del principio del placer son originarios, y congénitos de aquello que precisamente transgreden ${ }^{21}$.

Considerando esta cita, no sería descaminado recordar que también en Freud y la escena de la escritura, y justamente remitiendo a su Introducción..., Derrida escribe que "[....] Los conceptos de différance y de retardo originarios se nos impusieron a partir de una lectura de Husserl"22. Pero esto no sólo es relevante en lo que concierne a Husserl, sino, a la vez, por lo que se señala acerca los estertores de este texto, de su escena de producción - por decirlo así. Derrida da cuenta que "Freud y la escena..." es un "fragmento de una conferencia pronunciada en el Institut de psychanalyse" y que "se trataba entonces de abrir un debate en torno a ciertas proposiciones expuestas en ensayos anteriores, especialmente en De la grammatologie (Critique, 223 y 224)"23. En las páginas de De la gramatología a las que se remite, se puede encontrar, justamente, que "el suplemento es peligroso en la medida en que nos amenaza de muerte" 24 , amenaza que unas pocas líneas más arriba tiene el nombre de "economía del suplemento" 25 . Poco antes, incluso, en un pasaje que es también un cruce, se afirma que "el espaciamiento como escritura es el devenir-ausente y el devenir-inconsciente del sujeto" y que "como relación del sujeto con su muerte, dicho devenir es la constitución de la subjetividad. En todos los niveles de organización de la vida, vale decir de la economía de la muerte"26. El sintagma economía de la muerte, encuentra otros puntos de localización y despliegue tanto en el referido

21 Derrida, Jacques, "Freud y la escena de escritura" en La escritura y la diferencia, Anthropos, Barcelona, 1989, p. 280.

22 Derrida alude a las últimas páginas de su "Introducción..." donde el retraso [retard] nombra "otro origen absoluto, anterior y posible en general", y una "alteridad del origen absoluto que aparece estructuralmente en mi Presente Viviente" y que comporta la "limitación fenomenológicas" (op. cit., pp. 161-162). Bajo este registro, en "La différance" Derrida señalará que "el privilegio concedido a la consciencia significa, pues, el privilegio concedido al presente" y que "la temporalidad transcendental de la consciencia es al «presente viviente» al que se concede el poder de síntesis y de concentración incesante de las marcas" (Márgenes de la filosofía, Cátedra, Madrid, 2008, p. 51). Respecto de aquella temporalidad trascendental, el retardamiento (Nachträglichkeit) impide "que se haga de la temporalización una simple complicación dialéctica del presente vivo como síntesis originaria e incesante, constantemente reconducida a sí" y "entramos en contacto no con horizontes de presentes modificados -pasados o por venir-, sino con un "pasado» que nunca ha sido presente y que no lo será jamás, cuyo «por-venir» nunca será la producción o la reproducción en la forma de la presencia" (Ibidem, p. 56).

23 DERrida, J., "Freud y la escena...", op. cit., p. 271.

24 DERridA, J. De la gramatología. op. cit. p. 198.

25 Idem.

26 Ibid., p. 89. El párrafo termina afirmando que "Todo grafema es de esencia testamentaria. Y la ausencia original del sujeto de la escritura es también la de la cosa o del referente." (Idem). Esto no está lejos del problema de lo viviente. Según escribe Derrida, "La escritura como pintura, pues, es a la vez el mal y el remedio dentro del fainesthai o dentro del eidos." (Ibidem, p. 367). Pero decíamos que el anterior pasaje es un cruce, pues el asunto del grafema testamentario invoca el mito de "Thot, el dios egipcio de la escritura evocado en el Fedro","el inventor de la astucia técnica, análogo a Hermes, ejercía también funciones esenciales en el rito funerario" (Ibídem, p. 89, n. 31). Sobre este punto, que remite al pensamiento del Platón en el Cratilo, y expone la semejanza entre la escritura y la pintura como zoografema alrededor del problema de la mimesis, Cfr. "La farmacia de Platón" en La diseminación, Fundamentos, Madrid, 2007, pp. 91-262. 
"Freud y la escena..." como en "La différance"27. Cierto lazo, pues, más allá del sintagma, se desata a partir de este nudo. Economía de la muerte, economía de la différance o economía del suplemento, como la interna exterioridad, se anudan ${ }^{28}$. Sólo de esta manera es que la différance podría construir la esencia de la vida, o podría darse pensar la vida como huella: como la muerte.

En este registro, La voz y el fenómeno y el rendimiento que moviliza la lectura de Valdemar de Edgar Allan Poe, tiene un lugar privilegiado. Jacob Rogozinski, ha apuntado que la elección del exergo de La voz y el fenómeno ("Como se recordará, le había preguntado si seguía durmiendo. Y ahora escuché: - Sí... No... Estuve durmiendo... y ahora... ahora... estoy muerto") no tiene nada de arbitrario y, más aun, que "se podría considerar este libro, donde él pone en juego la relación de su pensamiento con la fenomenología de Husserl, como una larga explicación con esta cita de Poe, con la paradoja que ella enuncia" ${ }^{29}$. Rogozinski, a continuación, vinculando y haciendo saltar el texto, se refiere al pasaje donde el narrador del relato dice que "Ernest Valdemar" era un "renombrado compilador de la Bibliotheca Forensica y autor (bajo el nom de plume de Issachar Marx) de las versiones polacas de Wallenstein y Gargantúa". Rogozinky acentúa este pasaje para indicar algo que nos parece de primer orden: "que Poe nos informe que su Valdemar suscribe sus escritos con el pseudónimo de 'Marx' (i!), no se podría dejar de sugerir que el cuento bien podría titularse ... Espectros de Marx" ${ }^{\prime 30}$.

El orden o la gramática de este vínculo, de este salto, no nos permite dejar pasar que en Espectros de Marx Derrida desliza dos objeciones al principio fenomenológico en general: "Dos conclusiones, pues: 1) la forma fenoménica del mundo mismo es espectral; 2) el ego fenomenológico (Yo, Tú, etc.) es un espectro. El faineszai mismo (antes de su determinación como fenómeno o como fantasía, como fantasma, pues) es la posibilidad misma del espectro, lleva la muerte, da muerte [...]"31. Estas dos conclusiones no podrían desatenderse considerando que aparecen en el último capítulo del libro, cuyo subtítulo

27 Así, lo que en "Freud y la escena.." aparece como la "economía de la muerte" en "La différance" se nombra como la "economía de la différance" (op. cit. pp. 40 y 54). Es muy importante que el movimiento de la différance, cuya "a" se mantiene secreta como una tumba (oikevis, escribe Derrida), señale la tumba de lo propio, la muerte del dinasta. Este asunto toca sin duda la dinámica de la majestad o de la soberanía, la potencia (dunamis) o el despliegue de la potencialidad del dinasta y de la dinastía. Así, más de tres décadas después, Derrida se referirá a una "Supervivencia [survivance]" en un sentido "que no es ni la vida ni la muerte pura y simple, ni pensable desde la oposición entre la vida y la muerte pura y simple, ni pensable desde la oposición entre vida y muerte", y subrayará que se trata de "una supervivencia que no está [...] por encima de la vida lo mismo que el soberano (superanus) por encima de todo" de "una supervivencia que no está más viva, ni, por lo demás, menos viva que la vida, ni más ni menos muerta que la muerte", de una "supervivencia [survivance] cuyo súper [sur] carece de superioridad, de altura, altitud o alteza, por consiguiente, de supremacía y soberanía" (DerridA, Jacques, $L a$ bestia y el soberano, op. cit., pp. 174-175).

28 "El afuera, exterioridad 'espacial' y 'objetiva' de la cual creemos saber qué es como la cosa más familiar del mundo, como la familiaridad en sí misma, no aparecería sin la grama, sin la diferencia como temporalización, sin la no-presencia de lo otro inscripta en el sentido del presente, sin la relación con la muerte como estructura concreta del presente viviente." (De la gramatología, op. cit., p. 92).

29 Rogozinski, Jacob,“Vie et mort(s) de M. Valdemar”, Alter. Revue de phénomenologie. № 8, 2000, p. 199.

30 Ibidem, p. 200.

31 Derrida, Jacques, Espectros de Marx, op. cit. p. 153. 
-“Aparición de lo inaparente. El 'escamoteo' fenomenológico"-carga inclusive con cierta insistencia del texto en general: la "no contemporaneidad a si del presente vivo" 32 . No habría que perder de vista, por lo demás, que es en ese último capítulo donde está la única referencia a La voz y el fenómeno. Cuando Derrida escribe que "el modo esencial de la presencia a sí del cogito sería el asedio", se refiere justamente a "la implicación extrañamente susurrada de la muerte (no sólo de un «yo soy mortal», sino de un "yo estoy muerto») en la declaración del «yo soy»"33.

Si como versa Borges "el muerto no es un muerto: es la muerte" ${ }^{34}$, se podría añadir, entonces, que la economía de la muerte indica la destitución del yo. Así, el "yo estoy muerto" de Valdemar vendría a ser la "muerte del yo". Toda esta "operación", sin embargo, es estratégica y no implica una inversión simple de la metafísica de la presencia, no se trata de una inversión de la primacía del logos, en virtud hacia la escritura entendida como reverso de la voz o de la "palabra viva" (del sujeto viviente, que liga esencialmente el Logos y la Vida). No se trata de una grafocentrismo. Recordemos que Derrida plantea que la desconstrucción debe pasar por una fase de inversión estratégica de las oposiciones: "Olvidar esta fase de inversión es olvidar la estructura conflictual y subordinante de la oposición. Significa pasar demasiado aprisa, sin detenerse sobre la oposición anterior, a una neutralización que, prácticamente, dejaría el campo anterior en su estado y se privaría de todo medio de intervenir efectivamente" ${ }^{35}$. En estos términos -lo que Derrida estrategia o estratagema- esto quiere decir que la economía de la muerte sólo se da a pensar como la complicación de la vida-la muerte. Así, inversión mediante, se trata de "sustraer la escritura a la devaluación que se hace en nombre de la presencia viva y plena, devaluación que ubica la escritura del artificio y de la muerte", de manera que pensar "la vida como escritura de la muerte, es [...] deshacer la determinación que constituye el presupuesto de fondo de la metafísica occidental: el ser como presencia"39.

32 Ibidem. p. 13.

33 Ibídem., p. 151, n. 4.

34 Borges, Jorge Luis, "Remordimiento por cualquier muerte" en Obras completas 1923-1972, Emecé, Buenos Aires, 1974, p. 33.

35 Derrida, Jacques, Posiciones, Editora Nacional, Madrid, 2002, p. 54

36 Goldschmit, Marc, Jacques Derrida, una introducción, Nueva visión, Buenos Aires, 2004.

37 "La afirmación de la vida no va sin el pensamiento de la muerte, sin la atención más vigilante, responsable, incluso asediada, obsesionada de este fin que no llega - a(1) llegar. Desde el momento en que hay una huella [trace], sea cual fuere, ésta implica la posibilidad de repetirse, de sobrevivir al instante y respecto a su trazado [et au sujet de son tracement], cuya muerte, desaparición, mortalidad al menos, atesta así. La huella figura siempre una muerte posible, firma la muerte. Por ello, la posibilidad, la inminencia de la muerte no es sólo una obsesión personal, es una manera de aceptar la necesidad de lo que se da a pensar, a saber, que no hay presencia sin huella ni huella sin desaparición posible del origen de dicha huella, por tanto, sin una muerte. Que, lo repito, no llega a(l) llegar, a(1) llegarme, que me llega, que llega a(l) no llegarme. Posibilidad de lo imposible" (Derrida, Jacques, "El otro es secreto porque es otro" en Papel máquina. La cinta de máquina de escribir y otras respuestas, Trotta, Madrid, 2003. p. 343).

38 Derrida, Jacques, Dar (el) tiempo, Paidós, Barcelona, 1995, p. 103.

39 Derrida, Jacques, Aprender a vivir por fin. Entrevista con Jean Birbaum, Buenos Aires, Amorrortu, 2006, p. 50. El asunto de la afirmación de la mayor intensidad de (la) vida se comunica, se toca, a la vez, con la afirmación de la intensa diferencia entre fidelidad-infidelidad. Como señala Derrida en una entrevista con Anne 


\section{Afirmación y plus de vida}

... la muerte está al alba porque todo ha comenzado por la repetición. Jacques Derrida, Elipsis.

En la lógica de la contaminación, pensar la afirmación la vida implica el pensamiento la economía de la muerte, del suplemento, de exceso ${ }^{37}$. Ahora bien, el exceso -surplusmuestra que la pura vida sería la muerte, la clausura sin por-venir y que la pura muerte no se distinguiría de la lógica del presente vivo. De esta manera la estratagema derridiana no trata una inversión simple, sino el espaciamiento de un entre. Pensar la economía de la muerte, entonces, convoca cierta afirmación del exceso de/en la vida.

Esto no quiere decir simplemente que la muerte o el/lo muerto sean los únicos que pueden dar. No, únicamente una 'vida' puede dar, pero una vida en la cual se presente y se deje desbordar esta economía de la muerte. Ni la muerte ni la vida inmortal pueden dar jamás, solamente una singular supervivencia [survivance] ${ }^{38}$.

Las sobrevida nombra, así, un espaciamiento que es un más que la vida y un más que la muerte. En este sentido, da pensar la afirmación de la mayor intensidad de (la) vida, la más tensa, la más intensa: "la supervivencia no es sólo lo que queda: es la vida más intensa posible" 39 . Esta intensidad es la que se hace lugar a partir de la muerte, la que se espacia como la afirmación incondicional del por-venir, pues, como escribe Gérard Bensussan, la positividad de la afirmación de la desconstrucción "resuena o consuena con la forma viviente de la vida [...] el acontecimiento propio de la vida, excesiva, desmesurada" ${ }^{40}$. En este registro, el exceso de la vida, su desmesura, nombra la mayor intensidad, es decir, la vida más que la vida. La apertura de la-vida-la-muerte, la promesa de una sobrevida, de esta manera, no se deja reducir al vivir o al morir sino que resta como la afirmación del porvenir: "Así, pues, la afirmación del por-venir: no es una tesis positiva. No es más que la afirmación misma, el «sí» en tanto es la condición de toda promesa o de toda esperanza, de toda espera, de toda performatividad, de toda apertura al porvenir" ${ }^{\prime 41}$. En este registro, la entrevista A corazón abierto (1998) resulta indicativa pues el cuasi-concepto de sobrevida se trama en torno a la cuestión del retraso y la mayor intensidad de vida:

Berger, de 1983: "No hay equilibrio, hay dos posturas tan locas la una como la otra; cada una, separadamente, es una especie de locura de muerte, y por lo tanto el deseo frenético que afirmo, es el de no renunciar ni a la una ni a la otra, porque ambas son mortales. La simple fidelidad pura es la muerte, tanto como la infidelidad. Entonces, se trata de una diferencia, la más tensa, la más intensa posible entre los dos extremos". ("Dialangues" en Points de suspension, Galilée, Paris, 1992, p. 141. Trad. nuestra).

40 Bensussan, Gérard, “"Oui, la survie...» Note sur le carré affirmatif de la déconstruction” en Rue Descartes, 2006/2 n 52, p. 5. Luego escribe: "El sí, y por supuesto su reafirmación en el soplido de «un sí, sí» [...] no hace son recitar «un compromiso que no tendrá lugar fuera de esta repetición de una performance sin presencia». Dice y describe performativamente, el acontecimiento propio de la vida, excesiva, desmesurada [...] El cuadro afirmativo de la desconstrucción, avant, dans, sans, presque, inscribe en el círculo del sí lo que Derrida llama «la vida más que la vida»" (pp. 57-60. Trad. nuestra).

41 Derrida, Jacques, Mal de archivo. Una impresión freudiana, Trotta, Madrid, 1997, p. 76. 
Si sólo existiese la tradición, el pasado, el cierre a todo lo que llega, sería la muerte. Si sólo hubiese lo que llega de nuevo y no conozco, lo que viene a mí sin que yo tenga la más mínima capacidad de responder o de prepararme para ello, también sería la muerte. En cualquier caso, a lo que resisto es a la muerte. Elijo, por lo tanto, no la vida (en el sentido biológico) a cualquier precio, sino-digamos- la mayor intensidad de vida posible en cada momento [...] la cuestión del retraso siempre me ha tenido ocupado y no opondré el sobrevivir a la muerte. He llegado incluso a definir el sobrevivir como una posibilidad diferente o ajena tanto a la muerte como a la vida, como un concepto original. El sobrevivir es un concepto que no se deriva. Hay supervivencia desde el momento en que hay huella [...] jamás pude pensar el pensamiento de la muerte o la atención a la muerte, incluso la espera o la angustia de la muerte como algo distinto de la afirmación de la vida. Se trata de dos movimientos que, para mí, son inseparables: una atención en todo momento a la inminencia de la muerte no es necesariamente triste, negativa o mortifera, sino por el contrario, para mí, la vida misma, la mayor intensidad de vida ${ }^{42}$.

Se podría colegir que la sobrevida guarda y expone cierta insistencia en la textualidad Derridiana. En efecto, como escribe Nancy, "Jacques Derrida hizo del «sobrevivir» un concepto" "43, o más bien, un cuasi-concepto, pues en el registro de la huella, registro que comporta cierta an-arkhía, el cuasi-concepto de sobrevida no se deriva. De ahí, por ejemplo, es que deteniéndose en una encadenamiento que involucra tanto al hospes, como al hostis, hostage, host, guest, ghost, holy, ghost y el Geist, es que Derrida, en Aporias, se inclina "a considerar el duelo [dueil] y la (re)aparición [revenance], la espectralidad o la super-vivencia [survie], como unas categorías no derivables o como unos derivados no reductibles" ${ }^{\prime 4}$. La sobrevida, como el suplemento, es originaria ${ }^{45}$. No se deriva de ninguna naturaleza, identidad, pertenencia o finalidad anterior, no se deriva de la plenitud de la presencia ni del presente viviente. Es decir, si en el proceso de la conciencia interna del tiempo "el presente no aparece ni como la ruptura ni como el efecto de un pasado, sino como retención de un presente pasado, es decir, como retención de retención, etcétera"

42 Derrida, Jacques, “A corazón abierto” en ¡Palabra!, instantáneas filosóficas, Trotta, Madrid, 2001, pp. 19-41. (Las cursivas son nuestras)

43 NANCy, Jean-Luc, El intruso, Amorrortu, Buenos Aires, 2006, p. 48.

44 Derrida, Jacques, Aporías: morir esperarse (en) los límites de la verdad, Paidós, Barcelona, 1998, p. 102. Poco antes, escribe que "la sobrevida" estructura "cada instante en una especie de torsión irreductible, la de una anticipación retrospectiva que introduce el a-contratiempo y lo póstumo en lo más vivo del presente vivo" (p. 94).

45 Véase el último capítulo de La voz y el fenómeno, "El suplemento de origen". Es en esta lógica del suplemento y del excedente, que entendemos que "El enunciado «yo estoy vivo» acompaña mi estar-muerto y su posibilidad requiere la posibilidad de que esté muerto; e inversamente. No es esto una historia extraordinaria de Poe, sino la historia ordinaria del lenguaje. Más arriba accedíamos al «yo soy mortal» a partir del «yo soy». Aquí entendemos el «yo soy» a partir del «yo estoy muerto» [... ] Pero este aparecer del Ideal como diferencia infinita no puede producirse más que en una relación con la muerte en general. Sólo una relación con mi-muerte puede hacer aparecer la diferencia infinita de la presencia. Al mismo tiempo, comparada con la idealidad de lo infinito positivo, esta relación con mi-muerte llega a ser accidente de la empiricidad finita. El aparecer de la diferencia infinita es finito él mismo. Desde ese momento, la diferencia, que no es nada fuera de esta relación, llega a ser la finitud de la vida como relación esencial consigo como con su muerte. La diferencia infinita es finita. No se la puede pensar, pues, ya, en la oposición de la finitud y de la infinitud, de la ausencia y de la presencia, de la negación y de la afirmación.” (pp. 158-165). 
y la retención "pertenece también esencialmente a la forma general de la protención que está, a su vez, comprendida bajo la forma absolutamente única y universal del Presente Viviente" , la temporalidad espectral de la sobrevida no podría tener la tesitura de un ahora comprendido como la retención de un presente-pasado y la protención de un presentefuturo, sino del imposible por-venir. No es descaminado, bajo este prisma sostener, como escribe Michael Naas, que Derrida no ha cesado de hablar de esta contaminación "de la vida y sus otros, esta partición [partage] entre, por ejemplo, la presencia viviente y su repetición, la palabra viva y la letra muerta, la vida y la sobrevida, el ser viviente y el espectro" ${ }^{47}$. La superficie del reparto, que tiene la síncopa de la reaparición de la sobrevida -es decir, lo que intentamos aventurar bajo la figura de la insistencia- no queda simplemente indecidida sino que resta como lo indecidible mismo, de manera que la superficie contaminante asesta un golpe interno a cualquier pre-valencia de la tradición fenomenológica por la autenticidad, por la propiedad del eigentlich o la prevalencia de cualquier arhké y, por lo tanto, sacude cualquier jerarquía o valorización jerarquizante. En la partición del vivir y del presente, es decir, la divisibilidad de la juntura o de la ocurrencia se da un tiempo radicalmente disyunto ${ }^{48}$.

Síncopa de la sobrevida o ritmo de cierta insistencia, pues en su última entrevista, concedida a Jean Birnbaum el 2004, Derrida in-cita esta afirmación de la vida y la preferencia por la sobrevida, en una operación que, aprés coup, la articula en una cadena cuasi-conceptual que complica la lógica de la posición u oposicionalidad y la traslada hacia los contornos de la huella y lo espectral.

Como ya he recordado, desde el principio, y mucho antes de las experiencias de supervivencia [survivance] que hoy me son propias, señalé que la supervivencia [survie] es un concepto original, que constituye la estructura misma de aquello que llamamos existencia [...] Somos estructuralmente supervivientes [survivants], marcados por esta estructura de la huella, del testamento. Pero, dicho esto, no quisiera dar crédito a la interpretación de que la supervivencia [survivance] está más del lado de la muerte, del pasado, que de la vida y el futuro [avenir]. No, la deconstrucción está siempre del lado del sí, de la afirmación de la vida. Todo lo que digo -al menos, desde 'Pas' en Parages-de la supervivencia [survie] como complicación [complication] de la oposición vida/muerte procede en mí de una afirmación incondicional de la vida. La supervivencia [survivance] es la vida más allá de la vida [la vie au-delà de la vie], la vida más que la vida [la vie plus que la vie], y el discurso que pronuncio no es un discurso mortífero; al contrario, es la afirmación de un viviente [vivant] que prefiere el vivir, y por tanto el sobrevivir [survivre], a la muerte, pues la supervivencia [survie] no es sólo lo que queda: es la vida más intensa posible [la vie la plus intense possible $]^{49}$.

\footnotetext{
46 DERRIDA, Jacques, Introducción... op. cit., p. 50.

47 NaAs, Michel, "Une ligne de vie" en VVAA, Derrida pour les temps à venir, Editions Stock, Paris, 2007, p. 63. 48 Cfr. Derrida, Jacques, Espectros de Marx, op. cit, p. 31.
} 
Toda la cuestión de economía de la muerte está, entonces, del lado del sí, de la afirmación incondicional y, por lo tanto, del porvenir como la posibilidad de lo (im)posible ${ }^{50}$. La complicación de la oposición vida/muerte, y la afirmación incondicional de la sobrevida es la vie au-delà de la vie y la vie plus que la vie, es decir, la vie la plus intense posible. La vida, así, está atravesada por la "afirmación incondicional que atraviesa a la deconstrucción" ${ }^{51}$. Esta "la afirmación invencible de un porvenir imprevisible", es la afirmación de "la experiencia del no-presente, del no-presente viviente en el presente viviente", es decir, de la experiencia "de lo super-viviente" ${ }^{52}$. Cuestión, como indicábamos, de intensidad y de insistencia ${ }^{53}$.

La afirmación, así, es la afirmación de la demora en lo más vivo de lo viviente, pero de manera tal que el máximo de vida, le plus de vie, está plegado a la finitud del no-más-vida o plus de vie ${ }^{54}$. En este sentido es que en La diseminación, Derrida se refiere a la "ambigüedad decisiva e indecidible del sintagma 'más de' ['plus de'] (suplemento y vacancia)"

49 Derrida, Jacques, Aprender a vivir por fin, op. cit, p. 49-50. (Las cursivas son nuestras). No podemos dejar pasar que Blanchot, en La lecture de Kafka, escribe: "Nosotros no morimos [...] somos esencialmente sobrevivientes [Nous ne mourons pas [...] nous sommes essentiellement des survivants]" (M. BLANCHOT, La part de feu, Éditions Gallimard, Paris, p.16). Sobre Derrida y Blanchot, Cfr. Billi, Noelia,“'Como si sobrevivir fuera importante...'. Muerte de Dios, literatura y política" (en Cragnolini, Mónica-comp. Entre Nietzsche y Derrida: vida, muerte, sobrevida, La Cebra, Lanús, pp. 239-261.) Por otra parte, Maximiliano Figueroa me ha recordado que George Simmel escribe acerca del más-vida (Mehr-Leben) y más-que-vida (Mehr-als-Leben). Al respecto, véase el libro de Simmel, Intuición de la vida (Buenos Aires, Terramar, 2004, pp. 22 y ss). Leyendo el primer capítulo de este libro, "La trascendencia de la vida", "Como para Simmel la vida es a la vez más vida [ plus de vie (mehr Leben)] et más que la vida [plus que la vie (mehr als Leben)], la cuestión radica en saber si el más-que-la-vida [plus-que-la-vie] prevalecerá en la oposición dialéctica con el más-vida [plus-de-vie], étant donné que ces deux éléments sont inhérents à la vie. Le danger est que la transcendance de la vie s'aliène dans des formes stables et autonomes sans vie" ("Georges Simmel et la culture féminine" en "Revue du Mauss 2002/1 (no 19, pp. 183-190). Nótese que la traducción alemana de citada última entrevista, vierte "Survie" como "Überleben" y justamente la expresión "la vie plus que la vie" como "mehr Leben als das Leben".

50 Respecto del porvenir, sería preciso no sólo remitir a los textos más recientes sino también, por ejemplo, a De la gramatología donde se señala: "El porvenir sólo puede anticiparse bajo la forma del peligro absoluto. Rompe absolutamente con la normalidad constituida, y por lo tanto, no puede anunciarse, presentarse, sino bajo el aspecto de la monstruosidad" (op. cit. p. 10); o a Elipsis, también de 1967: "en ese caso el tiempo de la escritura no sigue la línea de los presentes modificados. El porvenir no es un presente futuro, ayer no es un presente pasado" (La escritura y la diferencia. op cit., p. 409). Así, en esta clave, la cuestión de la escritura podría leerse bajo esta "figura". En La diseminación, en este sentido, se insiste en que "la escritura no se mantiene en ninguno de esos tiempos (presente, pasado o futuro en tanto que presentes modificados)" (op. cit. p., 13). Más adelante, incluso, en un pasaje que involucra a Mallarmé, podría leerse la operación de lo indecidible -en este caso, himen - como la puesta en escena de la desconstrucción de protención-retención del tiempo bajo el privilegio del presente: "Tal diferencia sin presencia o más bien desbarata el aparecer dislocando un tiempo ordenado al centro del presente. El presente no es ya una forma-madre en torno de la cual se distinguen y se reúnen el (presente) futuro y el (presente) pasado. Ni hay señaladas en ese himen entre el futuro (deseo) y el presente (cumplimiento), entre el pasado (recuerdo) y el presente (perpetración), entre el poder y el acto, etc., más que diferencias temporales sin presente central, sin un presente cuyo pasado y futuro no serían más que sus modificaciones." (ibidem, pp. 317-318).

51 Derrida, Jacques, "No la utopía, lo im-posible" en Papel Máquina, op. cit, p. 312.

52 Derrida, Jacques, "Marx e hijos" en Sprinker, Michael (comp.), Demarcaciones espectrales. En torno a Espectros de Marx, de Jacques Derrida, Akal, Madrid, 2002, p. 296.

53 Así, en Resistencias, escribe que "toda la deconstrucción es también una lógica de lo espectral y de la obsesión, de la supervivencia: ni presente ni ausente, ni vivo ni muerto" (DERRIDA, Jacques, Resistencias del psicoanálisis, Paidós, Buenos Aires, 1998, pp. 50-51). De igual manera, en otro lugar, confiesa: "Estoy obsesionado por la estructura sobreviviente" (Cfr. Peeters, Benoît, Derrida, Fondo de Cultura Económica Buenos Aires, p. 17). 
Si consideramos esta ambigüedad, en la signatura que le imprime la suplementaria vacancia de la différance, bien escribe Jean Grondin que el "plus de" es una expresión que "en francés tiene múltiples y a la vez contradictorios significados". Señala así: "1) plus de puede tener el sentido de una multiplicación, significando 'un gran número de' more, mehr, más, piú [...] 2) plus de, en efecto, también puede significar 'nada más' ['no más' 'plus du tout'], jsuficiente!"56. De igual manera, Delmiro Rocha escribe que "plus de vie" es "ambigua en francés porque la letra 's', que se puede leer o no dice a la vez el 'plus de vida' y 'no más vida" 57 . Así, cuando Derrida propone una sola definición -breve, elíptica, económica- para "desconstrucción" como "plus d'une langue", escribe que esa expresión indica un "más que un idioma y no más que un idioma"58. Del mismo modo, por referir sólo dos lugares de condensación, tal como "plus de sens" en Políticas de la amistad es traducido como "basta de sentido" 59 , el "Plus de métaphore" de "La Mitología blanca" que se traduce como "Más metáfora" bien podría trasladarse como "No hay metáfora" o, mejor, como "No-más-metáfora" 60 .

El plus de vida, así, concierta lo que, tempranamente, ya en La voz y el fenómeno se denominaba "una no-vida o una no-presencia o no-pertenencia a sí del presente viviente, una inextirpable no-originariedad" ${ }^{\prime}$. Se trata, entonces de un máximo de vida plegado a un no-más-vida. Siguiendo este rastro, la citada última entrevista no hace sino producir y volver sobre un despliegue anterior, no hace sino producir, en el registro de lo póstumo o de la anticipación retrospectiva. Se trata del texto Sobrevivir donde se hace lugar lo que aguanta [endurance] la intensidad de una "reafirmación (el sí, sí) de la vida, en donde el sí, que nada dice, solamente se describe a sí [qui ne dit rien, ne décrit rien que lui-même], a la escenificación [performance] de su propio suceso afirmativo [événement d'affirmation]", es decir, una acontecimiento afirmativo que "no existiría fuera de esta repetición de una escenificación sin presencia [performance sans présence]"62. De esta manera:

54 Cfr., Derrida, Jacques, Ecografías de la televisión, Op. cit., p. 54.

55 Derrida, Jacques, La diseminación, p. 411.

56 Grondin, Jean, "La définition derridienne de la déconstruction: Contribution au rapprochement de l'herméneutique et de la déconstruction" en Archives de Philosophie 62, 1999 pp. 8-9.

57 Rocha, Delmiro, Dinastías en desconstrucción. Leer a Derrida al hilo de la soberanía, Dykinson, Madrid, 2011, p. 126. Agrega: "Cabe recordar que la letra 's' que marca el plural, más allá de la polisemia, es el concepto de diseminación. Una vez que hay archivo, en este caso, una imagen registrada, por lo tanto, grabada y almacenada en un soporte que ya no somos nosotros, que ya no nos necesita, esta imagen anuncia la muerte y la vida después de la muerte. Una imagen seguirá siendo, más allá de la desaparición de lo fotografiado, la herencia viva de aquello que no es presente" (ídem).

58 Derrida, Jacques, Memorias para Paul de Man, Gedisa, Barcelona, 2008, p. 28.

59 Derrida, Jacques, Políticas de la amistad, Trotta, Madrid, 1998, p. 31.

60 Derrida, Jacques, Márgenes de la filosofía, op. cit., p. 259. Considérese esto también para "La doble sesión": "La diseminación de los blancos (no diremos de la blancura) produce una estructura topológica que circula infinitamente sobre sí misma mediante el suplemento incesante de una vuelta de más: más metáfora, más metonimia)" (La diseminación, p. 387). Considérese, pues, que aquel "plus de métaphore, plus de métonymie" puede traducirse como "no hay metáfora, ni metonimia".

61 J. DeRrida, La voz y el fenómeno, p. 43.

62 Derrida, J., "Sobrevivir: Lineas al borde", op. cit., p. 105. El Texto Sobrevivir: líneas al borde, que involucra "el himeneo o alianza con el lenguaje del otro" al conectar dos textos literarios, al hacer un cruce intensivo 
[...] sobrevivir puede significar una postergación o una trascendencia, la 'vida tras de la vida' o la muerte tras de la muerte; más vida o más que la vida y mejor; el estado de suspensión en el que está -y vuelve a estar nuevamente, y jamás habrás agotado la suspensión en sí misma [la survie peut être encore la vie ou plus et mieux que la vie, le suspens d'un plus-de-vie avec lequel nous n'en aurions jamais fini $]^{63}$.

El acontecimiento de la afirmación de la sobrevida ${ }^{64}$, hace aparecer toda la puesta en cuestión del presente. La sobrevida, plus-de-vie y plus-que-vie, puede ser incluso la vida, un más y mejor que la vida, es decir, el suspenso infinito de un arrêt de mort, el paso de un más o un plus de vida, de un más que la vida y de un no-más-vida, el paso (pasaje y no) respecto del cual no se terminaría jamás. De esta manera, en 1985, en una entrevista con James Creech, Peggy Kamuf y Jane Todd, afirma Derrida:

[...] no se trata de la sobrevivencia (survival) en el sentido de posteridad, sino de otro tipo de sobrevivencia, de "vivir más" [more living] [...] La sobrevida [survival] no es simplemente la vida después de la muerte, sino una extraña dimensión de "plus de vie" tanto "más vida" ["more life] como "no más vida" [no more life]. O un "plus de vie", que es "más que vida" [more than life]. Plus de vie y plus que vie . $^{6}$

La diseminación de la sobrevida como plus de vida, es, pues, más que presente: "El espacio de la diseminación no sólo pone al plural [pluriel] en efervescencia; se agita con la contradicción sin fin, señalada en la sintaxis indecidible del más [plus] ${ }^{1{ }^{\prime 6}}$. Es en esta gramática del suplemento, del resto, del excedente, que La diseminación refiere al "pluscuampresente" [plus-que-présent] y que en De la gramatología se precisa que el "suplemento [supplément] se añade [s'ajoute], es un excedente [surplus $]^{67}$. El "sur" de "survie",

entre "el par de pretextos que son The Triumph of Life y L'arrêt de mort" (p. 81), compone la puesta en escena la cuestión de la sobrevida alrededor de la escritura de SHELLEy y Blanchot, pues la vida y el triunfo de la vida se trasladan en torno a la pena de muerte y/o sentencia de muerte. En este caso, el encuentro de un texto con otro (y por otro), se podría traducir como la juntura entre el suspenso de la muerte y la afirmación de la vida: "la lucha entre vida y muerte como light y nigth, es elemento de Triumph of life así como de L'arrêt de mort" (Ibidem, p. 167).

63 Ibídem, pp. 79-81.

64 Comentando la relación esencial entre Überleben y Übersetzen, y Fortleben (sobrevida en el sentido la continuación de la vida y no como vida post-morten), Derrida escribe: "La supervivencia [survie] de las obras, no de los autores. Quizá la supervivencia [survie] de los nombres de los autores y de las firmas, pero no de los nombres. Tal Supervivencia [survie] da una plus de vida [plus de vie], más que un sobrevivir [survivance]. La obra no sólo vive más tiempo, vive más y mejor [plus et mieux], por encima de los medios de su autor" (DERRIDA, J., 'Torres de Babel' 449-450).

65 Derrida, Jacques, "Deconstruction in America: an interview with Jacques Derrida" en Critical exchange \#17: winter, 1985, p. 25. (La traducción nuestra). En "Fe y saber", refiriéndose a "la marioneta" en tanto "máquina muerta y más que viviente [machine morte et plus que vivante]", como "la fantasmagoría espectral [phantasme spectral] del muerto como principio de vida y de supervivencia [mort comme principe de vie et de sur-vie]", Derrida va a señalar que su "principio mecánico aparentemente es muy simple: la vida no vale absolutamente salvo si vale más que la vida [plus que la vie], por consiguiente, salvo si lleva luto por sí misma, salvo si se convierte en lo que es en el trabajo del duelo infinito, en la indemnización de una espectralidad sin bordes" ( $E l$ siglo y el perdón, seguido de Fe y saber Buenos Aires, Ediciones de la Flor, 2003, p. 104).

66 Derrida, Jacques, La diseminación., op. cit., p. 66. Más adelante: "Con algún suplemento, nuestras preguntas no tendrán ya que nombrar más que la textura del texto, la lectura y la escritura, el dominio y el juego, las paradojas de la suplementariedad, así como las relaciones gráficas de lo vivo y lo muerto” (p. 95). 
indica, así, el "surplus" de la vida, el excedente del presente viviente, un "exceso, que en la vida triunfa sobre la vida y en el tiempo vale más que la eternidad de la vida, es ya totalmente diferente de la vida o de la eternidad de la vida" ${ }^{68}$, es decir, "un exceso de vida que resiste al aniquilamiento" ${ }^{\circ}$. Bajo la anagramática del plus, que se impulsa de punta a cabo hasta su última entrevista, y hasta su última oración, el pensamiento en Derrida no es sino afirmación -alegría y paso de danza- del porvenir de la vida. Se trata, así, de "un paso más allá de la vida [passage au-delà de la vie]"70, de cierta "convertibilidad de la vida y de la muerte, de la presencia y de la ausencia" que promete "la espectralidad testamentaria [revenance testamentaire] de un más-que-vida [plus-de-vie], de un sobrevivir [survivre]" $]$. En este sentido, la afirmación de la promesa de cierta "alegría" que "ligada a la acción de la muerte", "no pertenece a la economía de la vida" 72 sino a la "sobre-verdad del sobre-vivir" que radica en "la calidad hipertópica de este proceso" según el cual "la Cosa se da sin darse" y "tiene lugar sin tener lugar" 73 .

Alegría [Gaieté], reafirmación, triunfo (del) sobre : sobre la vida y de la vida, sobrevida, simultáneamente entre la vida y la muerte en la cripta, plus-que-vie, plus-de-vie, aplazamiento [sursis] e hípervitalidad, suplemento de vida que es mejor que la vida y la muerte, triunfo de la vida y de la muerte. Sobrevida que es mejor que la verdad y que sería, al menos, la Cosa por excelencia: sobre-verdad ${ }^{74}$.

Lo hipertópico, es lo in-ubicado y sobre-ubicado. La ubicuidad que sobrevive "como un fantasma o una aparición" 75 , no podría confundirse con la lógica del signo y del significado trascendental. Desbarata, más bien, la intuición de la cosa 'misma', de la cosa en carne y hueso, libre de toda entame, libre de toda encentadura. Así, tal como el texto de Derrida se inclina a leer L'arrêt de mort como un como un fascinante tratamiento de la verdad, y por lo tanto se inclina a leer este relato como un "relato anteriormente sin relato [" récit » sans récit]", es que tendríamos que acoger la huella suplementaria de "la sobre-verdad [survérité], en el complemento [supplément] de la verdad sin verdad [vérité sans vérité]"76**

67 Cfr., Derrida, Jacques, La diseminación, op. cit., pp. 462 y ss. Así también, en Posiciones: “[...] la falta y el excedente [surplus] no pueden nunca estabilizarse en la plenitud de una forma o de una ecuación, en la correspondencia inmóvil de una simetría o de una homología." (op. cit. p. 45.). También en estos términos, pero alrededor de Levinas, piensa Derrida el Adiós : "Le surplus d'un infini de sens, le plus-de-sens à l'infini, $l$ ' à-Dieu en témoigne, sans doute, mais, si je puis dire, à l'heure de la mort. Et d'une mort qu'il ne faut plus approcher selon l'alternative de l'être et du néant. Alors, à l'heure de cette mort, le salut ou l'appel se disent à-Dieu." (Adieu à Emmanuel Lévinas Galilée, Paris, 1997, pp. 207)

68 DERRIDA, Jacques, "Sobrevivir..." op. cit, p. 122-12.

69 Derrida, Jacques, Mal de archivo. op. cit. p. 67.

70 Derrida, J. Políticas de la amistad, op. cit, p. 20.

71 Ídem.

72 DerRida, Jacques, "De la economía restringida a la economía general (Un hegelianismo sin reserva)" en $L a$ escritura y la diferencia, op. cit. p. 355.

73 Derrida, Jacques, "Sobrevivir..." op cit. p. 135.

74 Idem. (Traducción modificada). Cfr. Vitale, Francesco, "Living On: The Absolute Performative" en Senatore, Mauro (Ed) Performatives after Deconstruction, BloomsBury, pp. 131-145.

75 Ídem.

76 Ibídem, p. 137.

* Artículo recibido: 27 de mayo de 2015. Aceptado: 21 de julio de 2015. 